\title{
PLIF TEMPERATURE AND VELOCITY DISTRIBUTIONS IN LAMINAR HYPERSONIC FLAT-PLATE FLOW
}

\author{
S. O'Byrne*, P.M. Danehy ${ }^{\dagger}$ \\ NASA Langley Research Center, Hampton, VA 23681 \\ and A.F.P. Houwing ${ }^{\ddagger}$ \\ Australian National University, Canberra, Australia 0200
}

\begin{abstract}
Rotational temperature and velocity distributions have been measured across a hypersonic laminar flat-plate boundary layer, using planar laser-induced fluorescence. The measurements are compared to a finite-volume computation and a first-order boundary layer computation, assuming local similarity. Both computations produced similar temperature distributions and nearly identical velocity distributions. The disagreement between calculations is ascribed to the similarity solution not accounting for leading-edge displacement effects. The velocity measurements agreed to within the measurement uncertainty of $2 \%$ with both calculated distributions. The peak measured temperature was $200 \mathrm{~K}$ lower than the computed values. This discrepancy is tentatively ascribed to vibrational relaxation in the boundary layer.
\end{abstract}

\section{INTRODUCTION}

Laminar boundary layers constitute an important test case for studying the effects of thermal and chemical nonequilibrium on hypersonic viscous flows. Being simpler than the more general case of turbulent and/or separated flow, they allow some uncoupling of thermal effects from the effects of fluid mixing. Moreover, the lower air densities and high Mach number conditions characteristic of hypersonic flight cause the boundary layer to remain laminar over greater distances than for subsonic flow, where laminar conditions are more the exception than the rule. A thorough understanding of thermal effects on hypersonic viscous flow is therefore necessary for the design of vehicles that can survive in the hostile hypersonic flight environment.

The literature contains several thorough investigations of thermal and chemical effects on hypersonic boundary layers, particularly for the relatively simple case of flat-

\footnotetext{
${ }^{*}$ NRC Postdoctoral Fellow, Hypersonic Airbreathing Propulsion Branch, MS168. AIAA member.

${ }^{\dagger}$ Research Scientist, Instrumentation Systems Development Branch, MS 236. AIAA member. ber.
}

plate flow, ${ }^{1,2}$ but the majority of these studies rely on surface measurements to infer behaviour in the boundary layer without directly measuring flowfield properties. Over the past three decades, non-intrusive laser-based diagnostics have been used with success to study supersonic and hypersonic viscous flows. ${ }^{3-5}$

The experiments described here are discussed in more detail in Ref. 6. The experiment was an extension of previous work discussed in Ref. 7, at a slightly higher Mach number and adding a flow tagging velocity measurement and a surface heat flux measurement to the temperature measurements performed in that study. Some of the velocity measurements described in this paper were presented in Ref. 8, but that work was concerned with the technique rather than its fluid-mechanical significance.

This paper is split into sections according to the techniques used for measuring or calculating the flow conditions. The free-piston shock tunnel facility used to generate the flow and the initial flow calibration experiments are discussed first. The important considerations for performing two-line planar laser-induced fluorescence (PLIF) temperature and velocity measurements will be presented with the results of each of the measurements. Particular attention is paid to quantifying the measurement uncertainties for both techniques. References 9 and 8 respectively contain a more general overview of the two-line PLIF thermometry and flow-tagging velocimetry techniques employed in this paper.

The remainder of the paper contains a comparison with experimental results of computed temperature and velocity distributions across the boundary layer. These distributions were obtained using the commercial CFD-FASTRAN CFD code and a first-order locally-similar boundary layer computation that accounts for the divergent freestream produced by the facility nozzle.

\section{FACILITY AND FLOWFIELD CONDITIONS}

The hypersonic freestream flow for these experiments was produced using the T2 free-piston shock tunnel. ${ }^{10} \mathrm{~T} 2$ is particularly well-suited to investigation of hypersonic flow using laser-induced fluorescence. The diameter of the conical nozzle exit is a relatively small $73.6 \mathrm{~mm}$, min- 
imising the systematic errors caused by absorption of the laser as it passes through the flow. The facility also has a turn-around time from one operation to the next of approximately 30 minutes. This allows a sufficiently large number of experiments to be performed to quantify measurement uncertainties.

The test gas for these experiments is a mixture of $98.9 \%$ nitrogen and $1.1 \%$ oxygen. When the shock reflects at the inlet of the facility's conical nozzle, the mixture reacts to form $1 \%$ nitric oxide, which is used to perform the PLIF experiments. This is a small enough amount to produce reasonable signal without significantly deviating in behaviour from a pure nitrogen flow. The reservoir conditions for the tunnel are calculated using the equilibrium shock tube code ESTC, ${ }^{11}$ based upon measured nozzle reservoir pressure and shock speed. These reservoir conditions are then used to calculate the freestream conditions using STUBE, ${ }^{12}$ an inviscid, one-dimensional nozzle code that accounts for finite-rate chemistry. Vibrational freezing is also accounted for using a sudden-freezing model. The calculated reservoir conditions were $p_{0}=27.5 \pm 1.4 \mathrm{MPa}$ and $T_{0}=4590 \pm 180 \mathrm{~K}$, corresponding to a stagnation enthalpy of $5.8 \pm 0.2 \mathrm{MJ} / \mathrm{kg}$.

Axial and radial distributions of pitot pressure were obtained using a piezoelectric pressure transducer (Piezotronics PCB type 113M165), with each measurement occurring on a separate tunnel run. Radial pitot distributions indicated uniform conditions in the nozzle core flow. An iterative calculation using the Rayleigh supersonic pitot formula was used to determine the effective nozzle expansion angle once the boundary layer was accounted for. For the geometrical conical nozzle angle of $7.5^{\circ}$, the pitot survey indicates an effective expansion angle between $6.5^{\circ}$ and $7^{\circ}$. Nominal calculated freestream quantities are $p_{\infty}=$ $2.4 \pm 0.2 \mathrm{kPa}, T_{\infty}=360 \pm 25 \mathrm{~K}, u_{\infty}=3185 \pm 80 \mathrm{~m} / \mathrm{s}$, $M_{\infty}=8.6 \pm 0.15$. The uncertainties are estimated based upon the uncertainties in the nozzle expansion angle, the measured tunnel shock speed and reservoir pressure. Values of each parameter that varied by one standard deviation were used in the codes to estimate the uncertainty of the computed freestream and reservoir quantities. The quoted uncertainties do not account for systematic errors due to possible inadequate modelling of chemical or vibrational processes or attenuation of the shock speed as the shock propagates through the test gas. The speed, measured using two transducers separated by $1270 \mathrm{~mm}$, is an average speed for the shock compression process.

The rectangular flat-plate model used in these experiments is shown in Fig. 1. The leading edge was sharp, having a diameter of $90 \pm 20 \mu \mathrm{m}$, and the underside of the model consisted of a $5^{\circ}$ wedge. The plate had a chord and span of $120 \mathrm{~mm}$ and $50 \mathrm{~mm}$ respectively.

The plate was mounted in the test section using a sting, and placed so the flat surface of the plate lay along the nozzle axis. The sting was aligned with the $\mathrm{T} 2$ nozzle axis using a helium-neon laser. Apertures of $2 \mathrm{~mm}$ diameter were placed at either end of the sting holder and in the nozzle.

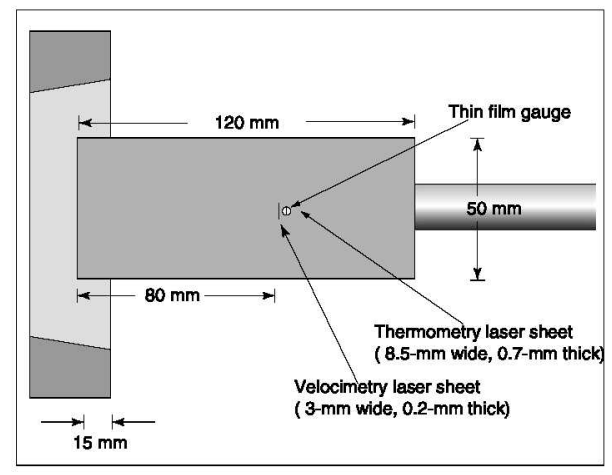

Figure 1: Plan view of flat-plate model showing measurement location for temperature and velocity measurements.

The sting was tilted and translated until the laser passed through the nozzle throat. It is estimated that this method of alignment minimises the inclination of the model, and the angle of incidence was less than $0.5^{\circ}$ from zero incidence. It is important to ensure that the freestream is not at incidence, because the measured temperature and velocity profiles will be sensitive to any misalignment. The effect of possible misalignment on the measured temperature distribution is significant and will be discussed in the analysis of results.

All measurements of velocity and temperature were obtained in a small region centered at a point $80 \mathrm{~mm}$ downstream of the plate's leading edge. This particular measurement position was chosen for two reasons. The measurements are obtained far enough from the leading edge for hypersonic displacement effects to be negligible, but close enough to the leading edge for disturbances arising from the corners of the plate not to intersect until well downstream of the measurement point. For the freestream Mach number of 8.5, these disturbances would intersect at a point $210 \mathrm{~mm}$ downstream of the plate leading edge. The leading edge of the plate was placed $15 \mathrm{~mm}$ upstream of the nozzle exit, so that the measurement point could be accessed through the test section window.

A convenient indicator of the effect of the leading edge upon the boundary layer flow is the hypersonic viscousinteraction parameter, $\chi=\left(M_{\infty}^{3} \sqrt{C_{e}}\right) / \sqrt{R e_{x}}$, where $C_{e}$ is the Chapman-Rubesin parameter. When $\chi \gg 1$, viscous interactions are important, which is the case near the flat-plate leading edge. Leading-edge effects are considered small when $\chi \leq 1$. At the measurement point for these experiments, $\chi \simeq 1.1$, so viscous interactions are not expected to be significant.

The Reynolds number $R e_{x}$ at the measurement point is $2.2 \times 10^{5}$. This is well below the transition Reynolds number of $10^{6}$ measured in Ref. 13 for flat-plate flows in the T4 free-piston shock tunnel facility, a similar facility but much larger than the one used in these experiments. The boundary layer is therefore expected to be laminar.

As can be seen from Fig. 1, a thin-film gauge was 


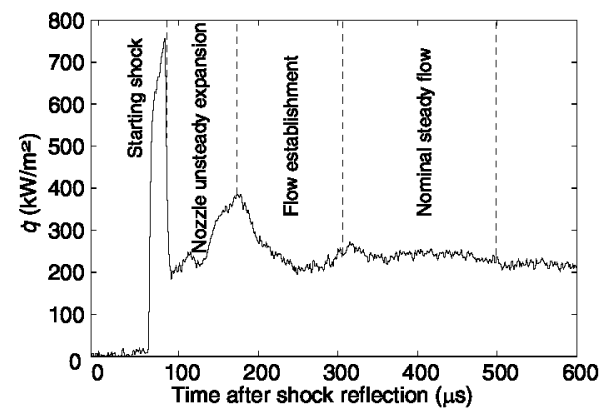

Figure 2: Calculated heat flux distribution.

placed $2 \mathrm{~mm}$ downstream of the measurement point, that is, $82 \mathrm{~mm}$ downstream of the leading edge. This gauge consisted of a $0.1-\mu \mathrm{m}$ thick, $0.3-\mathrm{mm}$ wide and $1-\mathrm{mm}$ long film of nickel that was vapour-deposited on a cylindrical quartz rod. The rod was 4-mm long and had a 2.1-mm diameter. The thin film gauge formed one arm of a balanced Wheatstone bridge circuit and the temperature response was calibrated by comparison with a type-K thermocouple in a variable-temperature water bath. The variation of heat flux during a tunnel run was calculated from the measured temperature using a one-dimensional heat transfer analysis. ${ }^{14}$ The temperature plots were smoothed using a $15-\mu$ s running average before differentiating to determine heat flux.

Figure 2 shows a typical calculated heat transfer trace. The gauges showed the same general features as described in previous free-piston shock tunnel investigations of flat plate heat flux measurements in Refs. 1 and 2.The heat flux reaches a constant value of $240 \mathrm{~kW} / \mathrm{m}^{2}$ from approximately $320 \mu$ s after shock reflection. The shot-to-shot standard deviation of the measured heat flux was $30 \mathrm{~kW} / \mathrm{m}^{2}$. The flow phenomena causing the heat flux variations are also shown on the figure. The rightmost dashed line indicates the end of the test time based upon estimations of the arrival of driver gas in the test gas. The PLIF experiments were all performed $360 \mu$ s after shock reflection.

This heat flux can be compared to the theoretical expected heat flux for a flat plate in a compressible flow by calculating the Stanton number, $S t=q_{w} /\left(\rho_{e} u_{e}\left(h_{r}-h_{w}\right)\right)$, where the suffix $e$ refers to local conditions at the edge of the boundary layer, the suffix $w$ refers to conditions at the wall, and $h_{r}$ is the recovery enthalpy, defined by $h_{r}=h_{e}+\operatorname{Pr}^{\frac{1}{2}}\left(\frac{1}{2} u_{e}{ }^{2}\right)$. Using the nominal freestream values provided by STUBE and assuming a Prandtl number of 0.7, the calculated Stanton number is $(7.8 \pm 1.0) \times 10^{-4}$. According to Ref. 1, St can be calculated by using the reference enthalpy concept and the Reynolds analogy to give

$$
S t \sqrt{R_{L}}=0.332\left(P r^{\star}\right)^{-\frac{2}{3}}\left(\frac{\rho^{\star} \mu^{\star}}{\rho_{e} \mu_{e}}\right)^{\frac{1}{2}}
$$

where starred quantities are calculated at the reference enthalpy conditions. Using Equation 1 at the measurement point gives a value of $6.8 \times 10^{-4}$ for $S t$, which agrees within uncertainty with the measured value.

\section{MEASUREMENT SYSTEM ARRANGEMENT}

The PLIF system used for these measurements consisted of a Lambda Physik EMG150ETS XeCl excimer laser that pumps a Lambda Physik Scanmate II dye laser operating with coumarin 2 dye, whose output was doubled using a type I BBO crystal to provide 226-nm light, exciting the $A^{2} \Sigma^{+} \leftarrow X^{2} \Pi(0,0)$ vibrational band of nitric oxide. This system produces $3-6 \mathrm{~mJ}$ of $226-\mathrm{nm}$ radiation at the laser exit. Approximately $8 \%$ of this light is diverted to a pair of quartz diffusers and a calibrated UV-sensitive photodiode, to measure the average energy of each laser pulse. Another $8 \%$ is diverted and focussed in a fuel-rich hydrogenoxygen flame. This interaction region is imaged onto a McPherson 0.5-m spectrometer, which spectrally filters the fluorescence in the flame. A Hamamatsu R446 photomultiplier tube measures the fluorescence intensity. The output of this photomultiplier is processed using a gated integrator and used to perform fluorescence excitation scans as the dye laser frequency is varied. These scans are performed immediately prior to each facility run, to ensure that the laser is tuned to the correct wavelength, to minimise systematic error caused by run-to-run variations in laser wavelength.

The main portion of the beam travels through a prism periscope and is formed into a sheet. This sheet passes through the flow via a fused silica window in the test section, as shown in Fig. 3. A Princeton Instruments intensified CCD (ICCD) camera is located beneath the test section and captures the fluorescence. A 2-mm thick Schott UG5 filter is placed in front of the camera to filter out scattered light at the laser frequency and much of the broadband luminosity from the flow. The effect of luminosity is also reduced by using gating periods of $330 \mathrm{~ns}$ for thermometry measurements and $50 \mathrm{~ns}$ for velocity measurements. The filter cuts off the fluorescence from the $(0,0)$ vibrational band of nitric oxide (NO) and collects light from the higher vibrational bands. This reduces the systematic error in measured temperature due to radiative trapping. ${ }^{9}$

The camera image acquisition time is varied by delaying the laser pulse and camera gate electronically with respect to the reflection of the shock at the nozzle reservoir. One interesting feature of this system is that the same apparatus can be used to measure both temperature and velocity, with the only difference being a rotation of the sheet orientation by 90 degrees for velocimetry, and a change in the intensifier gating delay and duration. Calibration tests were performed to ensure that the response of both the ICCD camera and the photodiode were linear over the range of laser and fluorescence intensities used in this study.

As mentioned in the introduction, these experiments re-examine previous hypersonic boundary layer measurements in Ref. 7, but at slightly different freestream conditions. The PLIF system used to obtain the temperature distribution across the boundary layer is nearly identical to that used in the previous investigation, but there are two important differences between the two experiments. 


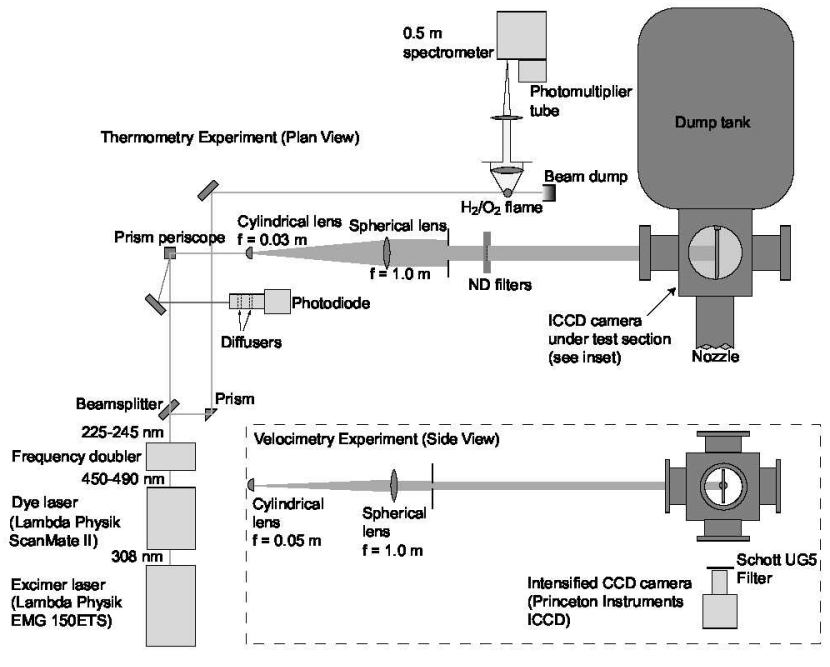

Figure 3: Laser system for thermometry and velocimetry experiments.

First, the nozzle throat was made smaller $(2.7 \mathrm{~mm})$ for this study. The reduction in the nozzle throat radius had the effect of reducing the freestream pressure, density and rotational temperature, making the boundary layer thicker. The second difference was that the telescope magnification system used in Ref. 7 was dispensed with and the camera was moved as close as possible to the test section, approximately $50 \mathrm{~cm}$ from the laser sheet. This had the disadvantage of reducing the magnification from 45 pixels $/ \mathrm{mm}$ in the previous study to $22.6 \pm 0.3$ pixels $/ \mathrm{mm}$. However, it was considered necessary to remove the possibility of chromatic aberration, and the lack of resolution was somewhat compensated for by the thicker boundary layer. Blurring of the broadband fluorescence due to chromatic aberration was considered responsible for the disagreement in the measured and computed boundary layer thicknesses in the previous investigation, so the ICCD camera used to image fluorescence in this study has an achromatic lens.

The assumption that chromatic aberration caused blurring in the experiments of Ref. 7 was based upon observations of blurring at the edge of the laser sheet by imaging PLIF from static nitric oxide in the test section. This blurring was also noticed in the present experiments, without the extra magnification optics, and was caused by diffraction of the laser sheet edge by the aperture used to define the edges of the laser sheet. When this aperture is placed $800 \mathrm{~mm}$ in front of the test section, the diffraction is noticeable when viewed with high magnification. An aperture placed inside the test section produced much less blurring at the edge of the laser sheet: across 4-5 pixels, or 180$220 \mu \mathrm{m}$. This amount of blurring is consistent with the ICCD camera's manufacturer-specified resolution, which is limited by the resolution of the intensifier and is 2 to 2.5 times smaller than that measured in Ref. 7 .

\section{ROTATIONAL THERMOMETRY}

The temperature measurements described here were performed using two-line PLIF thermometry. ${ }^{9,15}$ This technique has been used to measure temperatures in a variety of flow fields where large variations in temperature occur. The issues that must be addressed in order to make accurate two-line temperature measurements in shock tunnels are discussed in Ref. 7.

Temperature measurements were performed using two transitions in the $A^{2} \Sigma^{+} \leftarrow X^{2} \Pi(0,0)$ vibrational band of nitric oxide. Images were obtained on successive tunnel runs, using each of the transitions. The order in which images were obtained was randomised to reduce any temporal source of systematic error on the measurements. The two transitions chosen for this study were ${ }^{O} P_{12}(2.5)$ at 44069.4 $\mathrm{cm}^{-1}$ and ${ }^{P} P_{11}(35.5)$ at $44393.2 \mathrm{~cm}^{-1}$. These transitions were chosen for four main reasons. The transitions are isolated from other transitions, with less than $0.1 \%$ interference from other transitions at the freestream conditions. They are also not doublets, and so the ratio of fluorescence signals varies in a simple, monotonic manner over the temperature range of interest. The transitions were chosen to have similar line strengths so systematic errors due to saturation at high laser irradiances is minimised and they have excellent temperature sensitivity because of the large difference in their rotational energies $\left(\Delta F=2028 \mathrm{~cm}^{-1}\right)$. Such a large energy separation, $8 k T$ in the freestream and $2.7 k T$ at the peak temperature, implies a good temperature sensitivity throughout the flowfield, as described in Ref. 15. It is expected that shot-to-shot variations in the average signal have a relatively minor effect on the calculated temperature at the range of temperatures appropriate to these tests.

Because of the large energy separation, the signal for the ${ }^{P} P_{11}(35.5)$ transition was quite low in the freestream, approximately 200 counts above background or $0.3 \%$ of the total dynamic range of the camera. This necessitated the use of laser irradiances where saturation of the transition can no longer be considered negligible. The spectroscopic quantities of importance, average irradiance and the degree of saturation for the thermometry experiments are summarised in Table 1.

Six images were used at each of the transitions. Many more images were obtained, but only those images that showed uniform fluorescence were used for the thermometry measurements. The postulated causes and solution of the PLIF nonuniformity problem are discussed in Ref. 16. The images were corrected for background and flow luminosity by taking the average of 50 rows of the image which were outside of the laser sheet and subtracting this column from each of the columns in the experimental image. No luminosity was visible above the scatter in the background for the freestream, while the average scatter in the hottest part of the boundary layer was of the order of 10-20 counts, or $0.03 \%$ of the camera's dynamic range. Because no external laser-sheet profiling was performed, the twelve images were normalised to the laser profile in the freestream. 


\begin{tabular}{lcccccc}
\hline \hline Line & $F_{J}\left(\mathrm{~cm}^{-1}\right)$ & $G_{v}\left(\mathrm{~cm}^{-1}\right)$ & $B_{J^{\prime} J^{\prime \prime}}(\mathrm{cm} / \mathrm{J})$ & Irradiance $\left(\mathrm{W} / \mathrm{m}^{2}\right)$ & $\left(I / I_{\text {sat }}\right)_{e}$ & $\left(I / I_{\text {sat }}\right)_{\text {peak }}$ \\
\hline${ }^{O} P_{12}(2.5)$ & 73.58 & 948.66 & 146.99 & $7.51 \times 10^{8}$ & 0.22 & 0.31 \\
${ }^{P} P_{11}(35.5)$ & 2101.89 & 948.66 & 166.45 & $8.08 \times 10^{8}$ & 0.27 & 0.37 \\
\hline \hline
\end{tabular}

Table 1: Spectroscopic parameters, irradiance and degree of saturation for flat-plate temperature measurements. $F_{J}$ is the rotational energy, $G_{v}$ is the vibrational energy, $B_{J^{\prime}} J^{\prime \prime}$ is the Einstein absorption coefficient. The degree of saturation is presented external to the boundary layer $\left(I / I_{s a t}\right)_{e}$ and at the peak temperature within the boundary layer $\left(I / I_{\text {sat }}\right)_{\text {peak }}$. These values are calculated based upon measurement of saturation in a test cell at room temperature.

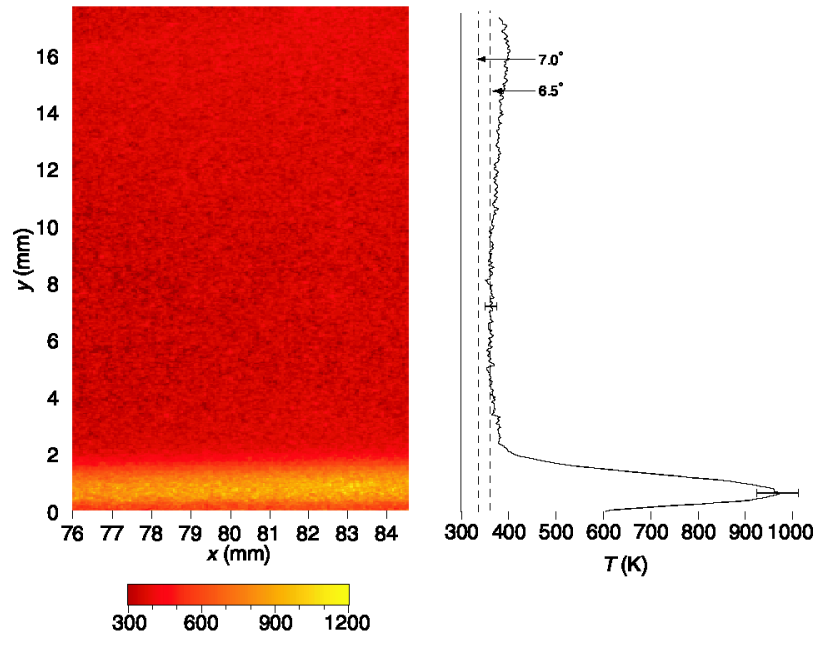

Figure 4: Temperature map and line plot for flat-plate measurements.

Profiling to the freestream implies that the conditions in the freestream are uniform, and any variation in the freestream PLIF signal is caused by a nonuniform laser-sheet profile. The laser sheet only extended $8 \mathrm{~mm}$ downstream, and changes in flow conditions over that distance should be small.

Once the raw data had been obtained, normalised, corrected for offset and averaged, the temperature was calculated using the two-line thermometry formula ${ }^{9}$

$$
T_{\text {rot }}=\frac{\left(F_{J_{2}^{\prime \prime}}-F_{J_{1}^{\prime \prime}}\right) / k}{\ln \left(\frac{E_{2} B_{2}\left(2 J_{2}^{\prime \prime}+1\right) \text { Sig }_{1}}{E_{1} B_{1}\left(2 J_{1}^{\prime \prime}+1\right) \text { Sig }_{2}}\right)}
$$

where $E_{1}$ and $E_{2}$ are the laser energies for the two transitions and the spectroscopic constants are provided in Table 1. The resulting temperature map is shown in Fig. 4, with a line plot obtained by averaging over 100 columns $(4.4 \mathrm{~mm})$ in the central part of the image. The averaging was sufficient to significantly improve the signal-to-noise ratio of the temperature distribution without measurably broadening the profile in comparison to a 100-pixel averaged distribution. The temperature distribution in the line plot increases from the freestream temperature across the leading-edge shock, then decreases again due to the expansion. The measured freestream temperature of $360 \pm 9 \mathrm{~K}$ agrees well with the STUBE calculation. At a distance of approximately $2.1 \mathrm{~mm}$ from the surface of the flat plate, the temperature begins to rise, reaching a peak temperature of $970 \mathrm{~K}, 0.8 \mathrm{~mm}$ from the surface of the plate. This temperature overshoot is characteristic of hypersonic cold-wall boundary layers. The wall cools the gas close to it, causing the temperature to peak within the boundary layer. A very gradual growth in the boundary layer is apparent in the flow direction, from the left to the right of the image. The estimated growth rate of the boundary layer, $d \delta / d x$ is $0.022 \pm 0.008$ over the visible region.

The upper portion of the temperature line plot shows an increase in temperature followed by a gradual decrease to the freestream temperature before reaching the boundary layer. The initial increase is caused by the leading-edge shock wave. The increase appears more gradual than expected from a shock wave because the data is averaged over 100 pixels, and the oblique shock is smeared. The decrease in temperature below the shock is caused by the expansion that turns the shocked flow parallel to the plate.

There are several sources of random uncertainty in the data used to calculate the temperature distributions. Uncertainties caused by the facility include shot-to-shot variations in shock tube shock speed, causing variations in all freestream flow conditions, variations in the NO mole fraction caused by processes such as entrainment of colder gas or driver gas and in the amount of soot absorbing the fluorescence signal. Uncertainties caused by the laser system include laser mode fluctuations, uncertainties in laser tuning relative to the peak of the absorption line and pulse-topulse variability of the laser irradiance.

Equation 2 is used as the basis for the uncertainty analysis, and it is assumed that the spectroscopic constants in that equation do not contribute to the uncertainty. The remaining sources of temperature uncertainty are the uncertainties in measured fluorescence intensity and laser irradiance. If the ratios of PLIF signal to laser energy are denoted by $R_{1}$ and $R_{2}$ for transitions 1 and 2 respectively, the uncertainty in measured temperature is given in terms of these ratios as ${ }^{6}$

$$
\Delta T=\sqrt{\left(\frac{\partial T}{\partial R_{1}} \Delta R_{1}\right)^{2}+\left(\frac{\partial T}{\partial R_{2}} \Delta R_{2}\right)^{2}}
$$

where $\Delta R_{1}$ and $\Delta R_{2}$ are the standard errors in the mean for $R_{1}$ and $R_{2}$ respectively.

The standard errors in the mean of the ratios of PLIF 
signal to laser energy, $R_{1}$ and $R_{2}$, varied from 9-13\%. Using Equation 3 results in a temperature uncertainty of $8 \mathrm{~K}(2.0 \%)$ in the freestream and $37 \mathrm{~K}(4.3 \%)$ at the peak temperature. Because of the low freestream signal for the ${ }^{P} P_{11}$ (35.5) images, the uncertainty in the background subtraction can have an effect on the measured temperature. Assuming an uncertainty of \pm 20 background counts causes a temperature uncertainty of $\pm 3.4 \%$ in the freestream and $\pm 0.2 \%$ at the peak temperature. Adding in quadrature the effects of fluctuations in background counts and uncertainty in the measured values of $R_{1}$ and $R_{2}$ gives a temperature uncertainty of $\pm 2.4 \%$ in the freestream and $\pm 4.5 \%$ at the peak temperature. These values are shown as error bars in Fig. 4.

It is impossible, given the available instrumentation, to separate each of the sources of random uncertainty in $R_{1}$ and $R_{2}$ from the others, or to be sure which contributions are most significant. The two most likely sources of uncertainty are fluctuations in the fraction of the laser energy in each of its longitudinal modes. ${ }^{17}$ These changes in turn change the degree of overlap between the laser and the transition from one shot to the next and manifest as variations in signal intensity. Another possible source of uncertainty is nonuniformity in the composition of the nozzle freestream, examined in Ref. 16. Measurements of PLIF signal variations in a static gas cell had a standard deviation of $\pm 15 \%$ at $2 \mathrm{kPa}$, compared to the measured standard deviation in the freestream of $\pm 23 \%$ for the ${ }^{O} P_{12}$ (2.5) transition and $\pm 29 \%$ for the ${ }^{P} P_{11}(35.5)$ transition. This suggests that a significant portion of the uncertainty could be due to the facility, but until independent measurements of the other possible sources of error are made, the main source of uncertainty cannot be isolated.

In addition to the random uncertainties discussed above, the three main sources of systematic error in the temperature measurements are: interference due to fluorescence from other transitions, saturation and laser sheet attenuation. The LINUS code ${ }^{17}$ was used to calculate the effects of laser sheet attenuation in the flat plate flowfield by using the measured temperature distribution, and assuming a constant pressure across the boundary layer. The code assumes a Gaussian laser frequency distribution and calculates the effect of absorption on this distribution as the laser propagates through the flow. It also accounts for the effect of saturation on the beam absorption. When both saturation and absorption are accounted for, the systematic errors are $+1 \mathrm{~K}$ in the freestream and $+2 \mathrm{~K}$ in the boundary layer. The effect of saturation partially cancels the effect of sheet attenuation and reduces its effect because saturation changes the absorption coefficient as the sheet propagates through the flowfield.

\section{VELOCIMETRY}

The velocimetry method is a straightforward time-offlight measurement method, and is outlined in detail in Ref. 8. Measuring velocity with this method involves ex- citing a nitric oxide transition using the same laser used for the thermometry experiments, waiting for a fixed time, then capturing the fluorescence using an ICCD camera with a gate duration which is much less than the delay time. In the time between the excitation of the gas and the opening of the camera gate, the excited, fluorescing fluid advects to a new position, which is recorded once the gate opens. Velocity is determined using a knowledge of the time delay used and a measurement of the displacement of the tagged region of fluid.

The laser apparatus is shown in Fig. 3 and the laser sheet orientation on the plate is shown in Fig. 1, with the measured dimensions of the sheet. The model was rotated by 1-2 degrees towards the camera, about the sting axis, so that the surface of the plate was only just visible. This was done to make the laser scatter easier to see. The rotation had a negligible effect on the measured boundary layer velocity profile and ensured that the zero-velocity flow at the plate surface could be used as a zero-displacement reference. The thickness of the laser sheet was chosen to be as close to the camera's minimum resolvable length as possible. The width of the sheet was chosen to be as large as possible, to maximise the captured fluorescence, but not so wide as to cause blurring of the image. The Princeton Instruments ICCD camera was operated at its minimum $f / 4.5$, which has a nominal depth-of-field of $2 \mathrm{~mm}$. The average energy in the laser sheet was approximately $1 \mathrm{~mJ}$, and approximately five times the saturation irradiance at the freestream conditions. Using a sheet is more effective than focussing a beam down to a spot because the signal is integrated along the sheet length, whereas the high degree of saturation produced by concentrating the laser as a beam would only increase the signal from that point by a much smaller amount.

The co-incidental overlap of the ${ }^{Q} Q_{22}(19.5)$ and ${ }^{Q} Q_{11}(12.5)$ transitions and their satellite transitions at $44227.71 \mathrm{~cm}^{-1}$ was used for the tagging measurements, because these four transitions have appreciable groundstate populations for the entire range of flow conditions throughout the boundary layer, and their strong transition probability ensures a strong fluorescence signal. Before each shot, the dump tank was filled with a 5-kPa mixture of $1 \% \mathrm{NO}$ in $\mathrm{N}_{2}$. This mixture was used to tune the laser to line centre and to obtain an image of the laser sheet in stationary flow. This image was used as a position reference, which could be subtracted from the displacement measured in the experiment, to remove any systematic error caused by the beam not being perpendicular to the flat plate.

Images of the flat-plate boundary layer were obtained using delays of $0,250,350,450,500,650$ and $750 \mathrm{~ns}$, on successive tunnel runs. Velocity profiles were obtained for all of these delay times. The images show a decrease in signalto-noise ratio as the delay increases. This is because of the exponential decay of the fluorescence during the delay time. After a 750-ns delay, for example, the fluorescence has decayed by $99.6 \%$.

The displacement profiles were obtained using Matlab 
routines that convolve each row of the image with a $3 \times 4$ pixel Gaussian function, oriented with the 3-pixel side in the streamwise direction, to smooth the data. The peak of the row is then determined and a third-order polynomial fitted to the three points closest to the maximum point, to determine the peak of the distribution. This process is repeated for each row of the image, to determine the displacement as a function of height above the plate.

Once the displacement had been measured, velocity was calculated using the method outlined in Ref. 8. For most of the images, velocity data was not obtained within $0.3 \mathrm{~mm}$ of the wall because of interference from laser scatter at the model surface, where the fitting algorithm would cause the peak of the distribution to jump to the position of maximum intensity at the wall and produce spurious measurements of zero velocity. This distance corresponds to the inner $15 \%$ of the boundary layer profile. These data were manually removed from the calculations. A better fitting algorithm may allow measurements to be made closer to the surface.

All nine velocity measurements were averaged to form a single profile. The velocity distribution in the boundary layer is typical for a laminar hypersonic boundary layer. The average freestream velocity is very uniform and has a value of $3035 \pm 60 \mathrm{~m} / \mathrm{s}$, when averaged over a $12-\mathrm{mm}$ region of the freestream. The standard deviation over that same range is $130 \mathrm{~m} / \mathrm{s}$.

The external velocity was also determined by plotting the displacement for each tunnel run, averaged over the $12-\mathrm{mm}$ region of the image external to the boundary layer, against the delay time. A straight line, passing through the origin, was used to fit the displacement data. The slope of this line determined $u_{e}$. Using this method results in a measured external velocity of $3086 \pm 60 \mathrm{~m} / \mathrm{s}$, which is in even better agreement with the nominal freestream velocity than the measurement obtained by averaging the individual velocity profiles.

Several sources of random uncertainty and systematic error have been accounted for in the quoted uncertainties of these velocity measurements. These effects are summarised in Ref. 8.

The boundary layer thickness based upon $0.99 u_{e}$ was measured to be $1.8 \pm 0.15 \mathrm{~mm}$. This corresponds to $\delta / x=0.023 \pm 0.002$, similar to the growth rate for the thermal boundary layer. Reference 18 derives a correlation for supersonic laminar boundary layer growth

$$
\frac{\delta}{x}=\left[5.0+\left(0.2+0.9 \frac{T_{w}}{T_{a w}}\right)(\gamma-1) M_{e}^{2}\right] \sqrt{\frac{C_{w}}{R e_{x}}}
$$

where $T_{a w}$ is the adiabatic wall temperature. This relation predicts $\delta / x=0.023$, although it was derived by assuming zero pressure gradient, so the measured boundary layer would be expected to be thicker. Another formulation for the boundary-layer thickness in the absence of a pressure gradient has been proposed by Ref. 2 . This relation is specific to hypersonic laminar boundary layers and takes the form

$$
\begin{aligned}
\frac{\delta}{x}= & 1.721 \sqrt{R_{x}} \times \\
& {\left[2.397+\frac{T_{w}}{T_{e}}+0.0965 \operatorname{Pr}^{1 / 2}(\gamma-1) M_{e}^{2}\right] }
\end{aligned}
$$

Substitution into this equation produces $\delta / x=0.017$. Thus the correlation underpredicts the boundary-layer thickness, presumably because it does not take the pressure gradient due to the nozzle into account.

Assuming that the conditions external to the boundary layer are equal to the values predicted by STUBE at the measurement position, i.e. that the pressure gradient parameter $\beta=0$, the method of Ref. 19 predicts $\delta / x=$ 0.0197 , compared with 0.0198 when the pressure gradient is included. The differences in velocity profile for diverging and non-diverging flow are predicted by the code to be very small. If this is the case, the prediction of Equation 5 may still be an underestimate of $\delta / x$. The boundary-layer growth rate as calculated using the method of Ref. 19 is just outside the quoted measurement uncertainty. The difference may be caused by the difference in predicted and measured freestream velocity. The comparison of measured and calculated profiles is presented in the next section.

Although laser-based measurements are usually considered nonintrusive, the irradiances used in the velocimetry experiments are large enough to measurably heat the surface of the flat plate. This can alter the velocity distribution by changing the plate surface temprature. To investigate the effect of laser heating, the laser irradiated a coaxial thermocouple mounted on a flat sheet of mild steel similar to the flat-plate model. The beam had a diameter just large enough to cover the thermocouple and the irradiance was adjusted to be equivalent to that used in the velocity measurements. The thermocouple registered a maximum temperature of $80 \mathrm{~K}$ above ambient, decaying to ambient temperature after $80 \mu \mathrm{s}$. Similarity calculations of boundary layer distributions for wall temperatures of 300 and $400 \mathrm{~K}$ show a difference of less than $1 \%$ in the velocity profile. The difference is small because the velocity distribution depends on the ratio of wall temperature to stagnation temperature, and the $100-\mathrm{K}$ change in plate surface temperature is only approximately $2 \%$ of the stagnation temperature.

The uncertainties achieved using the velocimetry method outlined in this paper are a significant improvement over previous measurements performed in free-piston shock tunnel facilities. Reference 20 contains measurements of the freestream velocity in a much higher-enthalpy flow, using both spark-tracer and magnetohydrodynamic techniques. The uncertainty in these measurements was $\pm 17 \%$. Ref. 3 used pitot pressure measurements and interferometric density measurements to infer a velocity profile in a hypersonic laminar boundary layer in the larger T3 freepiston shock tunnel facility. Those measurements produced an anomalous saddle-point in the velocity profile which is not expected in flat-plate boundary-layer profiles. These 
measurements show no such anomalies.

\section{COMPUTATIONS}

The flat-plate flowfield was calculated using CFDFASTRAN, a commercial pressure-based, finite-volume computational fluid dynamics (CFD) package. The main aim of performing this computation was to provide a comparison with the measured temperature and velocity distributions. The computation had to include as many of the features of the flow as possible, while converging quickly enough to be able to investigate the effect of varying important parameters. These competing criteria necessitated some compromises in the computations. The flow was assumed to be both chemically and vibrationally frozen, allowing a perfect-gas calculation to be used, as the reactingflow calculation was too computationally expensive. Certainly at such relatively low stagnation enthalpy, the flatplate flow should be chemically frozen. According to the vibrational relaxation rates of Millikan and White, ${ }^{21}$ the freestream should be vibrationally frozen, and the pressure throughout the boundary layer is low enough to assume vibrationally frozen flow throughout the computational domain.

The flat-plate boundary layer occurs within a diverging conical nozzle flow. This is a three-dimensional flowfield. The modelling of this three-dimensional flow was deemed too computationally demanding, so the divergence of the flow perpendicular to the plate had to be accounted for in a purely two-dimensional flowfield. This was achieved by making a Cartesian two-dimensional computational flowfield in which the area ratio varied in a manner identical to that of the axisymmetric nozzle flow. Comparison of the axial distribution of CFD-FASTRAN freestream flow properties with the axisymmetric STUBE computation showed agreement to within $2 \%$.

The computational domain was $120-\mathrm{mm}$ long and the top surface of the domain was made to diverge at a $5^{\circ}$ angle. The plate occupies $110 \mathrm{~mm}$ of the bottom boundary of the domain, with a 10-mm symmetry boundary between the inlet and the leading edge of the plate. This allowed the leading-edge shock to be formed within the computed flowfield. The grid cells were distributed linearly in the $x-$ direction and a power-law cell distribution was used in the $y$-direction. A Prandtl number of 0.7 and a Sutherland law temperature-viscosity relationship were assumed for these calculations.

Calculations typically required three to six hours of computing time for the residuals to converge by eight orders of magnitude, depending on the number of grid points in the calculation. A grid sensitivity analysis was performed to ensure adequate grid resolution. Three distributions were chosen: 100 points with an exponent of $1.5,100$ points with an exponent of 2 and 200 points with an exponent of 1.5. All three grid distributions produce nearly identical velocity profiles. With these results in mind, all subsequent calculations were performed using an inlet of 100 cells with
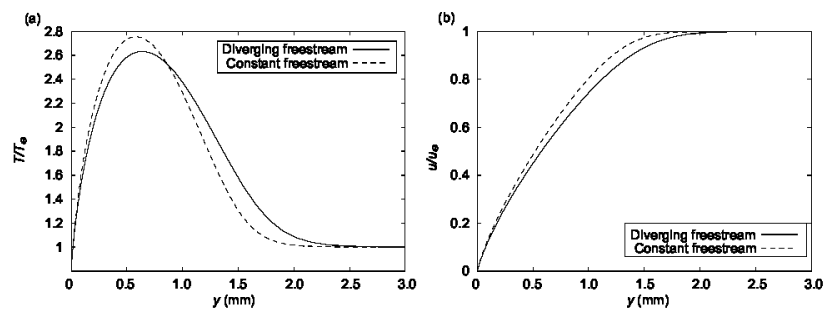

Figure 5: Comparison of computed (a) temperature and (b) velocity profiles assuming flow diverges at $6.5^{\circ}$ and flow with uniform freestream conditions.

an exponent of 2.0, to minimise computational time. This distribution has 29 grid points within the boundary layer at the measurement point, sufficient to adequately capture the boundary layer behaviour.

It is instructive to see the effect of flow divergence on the predicted distributions of temperature and pressure in the boundary layer, as it is sometimes assumed in experimental studies ${ }^{3,7}$ that the effect of divergence on the boundary layer is not significant. Figure 5 contains temperature and velocity distributions for the boundary layer assuming diverging and constant freestream conditions. The diverging conditions assume a conical nozzle divergence angle of $6.5^{\circ}$, while the constant conditions are those at the measurement point. Despite the fact that the freestream conditions are the same at both points, the diverging flow temperature and velocity profiles are approximately $10 \%$ thicker for the diverging freestream. This thickening of the temperature and velocity profiles is consistent with the theory of laminar boundary layers in a favourable pressure gradient. The peak temperature also decreases by approximately $10 \%$ for the diverging-flow case.

As mentioned in the velocimetry section, there was a worst-case difference of $150 \mathrm{~m} / \mathrm{s}$ between the average of the velocity profiles and the STUBE calculation. These two freestream velocities were used as inputs to the computation. The lower velocity value decreases the peak temperature by approximately $10 \%$ and increases the velocity and temperature boundary layer thicknesses by $8-10 \%$. Comparison with Fig. 5 indicates that the effects of flow divergence and an incorrect assumption of freestream velocity are similar. Thus, a failure to account for flow divergence may be misinterpreted as an effect of the assumption of the freestream velocity, or vice versa. This result shows the importance of having accurate measurements of both freestream velocity and temperature when comparing experimental and theoretical temperature distributions. Using the measured freestream velocity allows this source of ambiguity to be accounted for in any comparison with calculations.

The ratio of specific heats, $\gamma$, used for calculations can have a significant influence on the calculated flow properties within the boundary layer. The high temperatures and pressures in the nozzle reservoir generate chemically and thermally excited flow which can complicate any perfectgas calculation of the flowfield. The effect of thermal or 
chemical nonequilibrium is reflected in the value of $\gamma$ in a perfect-gas computation of the flow.

The STUBE program, which was used to calculate the temperature, pressure and velocity at the inlet to the CFDFASTRAN boundary layer calculation, is designed to account for both chemical and vibrational freezing in the nozzle. As the mixture in the nozzle is $98 \% \mathrm{~N}_{2}$, and the stagnation enthalpy is not high enough to cause significant dissociation, we can assume the flowfield to be diatomic and chemically frozen throughout. The gas is, however, in vibrational equilibrium in the reservoir. The vibrational temperature freezes inside the nozzle as the gas expands, because the frequency of molecular collisions becomes too low to maintain equilibrium of the vibrational energy. STUBE predicts vibrationally frozen flow in the freestream at the flat-plate condition. This prediction is supported by PLIF vibrational temperature measurements in Ref. 16 made in the same facility at a freestream condition having even higher pressure and lower stagnation enthalpy than this one.

Frozen flow can be shown ${ }^{22}$ to be isentropic, meaning that the flow can be treated as a perfect gas with a constant ratio of specific heats of $\gamma=1.4$. Fluid in vibrational equilibrium, on the other hand, has a ratio of specific heats closer to 1.3. This difference has a significant effect on the boundary layer temperature profile. The peak temperature when $\gamma_{f r}=1.4$ is approximately three times the freestream value, compared with an increase of 2.6 times for $\gamma_{f r}=1.3$. This is a significant difference, and larger than the uncertainty of the measurement technique. The velocity profiles are not much different in both cases, with the $\gamma_{f r}=1.4$ profile extending about $0.1 \mathrm{~mm}$ further than the $\gamma_{f r}=1.3$ profile.

A solution to the compressible boundary layer equations assuming local similarity was performed as a check of the CFD-FASTRAN calculation. The local similarity calculation used here is taken from Ref. 19. This implementation was chosen because it accounts for axial external-flow pressure gradients, which we have already shown to have a non-negligible effect on predicted temperature and velocity distributions. The similarity calculation was used to calculate temperature and velocity profiles, given the ratio of wall temperature to stagnation temperature, the freestream Mach number, the frozen ratio of specific heats $\gamma_{f r}$, the viscosity-temperature power-law constant $\omega=0.68$, the Prandtl number, $\operatorname{Pr}=0.7$ and the pressure gradient parameter, $\beta=0.252$.

The local similarity calculation produces profiles of dimensionless velocity $f^{\prime}$ and enthalpy ratio $g$, as a function of the dimensionless distance perpendicular to the plate, $\eta$. $\eta$ was transformed to the physical $y$-coordinate for comparison with experimental data and the CFD-FASTRAN computation.

The differences between the results of the two computations will be discussed in the next section for the boundary layer flow discussed here. In general, the CFD-FASTRAN and similarity calculations show the same trends when flowfield parameters are varied. Both calculations predict the same changes in temperature and velocity profiles as the divergence angle is changed. This fact was also noted when comparing local similarity and CFD calculations for different values of $\gamma_{f r}$. This is where the local similarity calculation can prove very useful. It produces profile shapes which are qualitatively accurate and correctly predicts trends when the important flow parameters are varied. Because the similarity calculation converges almost instantaneously on a standard Pentium desktop computer, it provides a quick and convenient way of testing the behaviour of the boundary layer.

\section{COMPARISON OF RESULTS}

Figure 6 (a) is a plot of the temperature profiles obtained when the calculations are performed assuming $\gamma_{f r}=1.4$. The profiles agree in a qualitative sense, in that the distributions all have the same form, but there is a significant disagreement in the peak temperature, with both calculations predicting a higher peak temperature than measured.

The agreement between the similarity calculation and the CFD-FASTRAN solution is good. The relative temperature jump in the boundary layer is nearly identical, with the difference in peak temperature evident in Fig. 6 due to the lower $T_{e}$ for the similarity calculation, which does not account for the leading-edge shock. The boundary layer is slightly thinner for the local similarity calculation. As for the discrepancy in peak temperature, the difference in profile width can be explained by the local similarity calculation not accounting for leading-edge displacement. Both calculated temperature profiles exhibit a noticeably thinner thermal boundary layer than the measured profile. As the error bars in the image show, the experimental uncertainties are not sufficient to explain these differences. Varying the nozzle expansion angle from $6.5^{\circ}$ to $7.0^{\circ}$ or changing the freestream velocity to the lowest measured value do not produce changes in the peak temperature of large enough magnitude to make the results agree to within the experimental uncertainty. Flow divergence was accounted for in the calculation and the previous explanation of blurring has been shown to be a small effect.

Examination of Fig. 6 (a) also shows a difference between experimental and calculated temperature near the wall. This is due to laser scatter from the wall interfering with the fluorescence signal, decreasing the signal ratio and predicting a higher temperature. For this reason, the temperature measurements in the $0.25-\mathrm{mm}$ region closest to the wall should be disregarded.

The only variable that makes the measured temperature distribution agree with the calculated value is $\gamma_{f r}$. If $\gamma_{f r}=1.3$, the agreement between measured and calculated temperature profiles becomes excellent, as can be seen from Fig. 7 (a). However, as already mentioned, the frozen value of $\gamma$ should be 1.4.

The agreement between the measured and calculated velocity profiles is very good, and within the measurement 
(a)

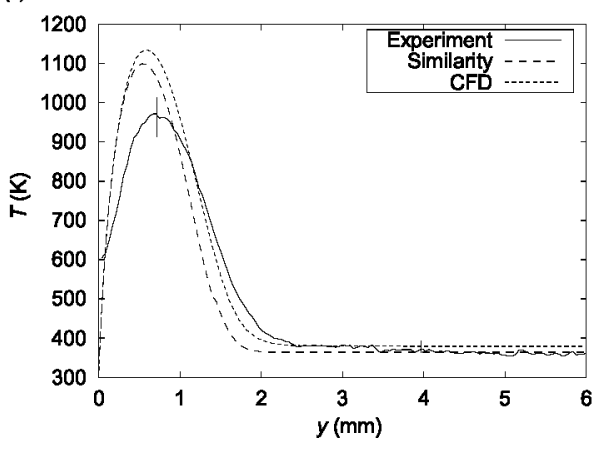

(b)

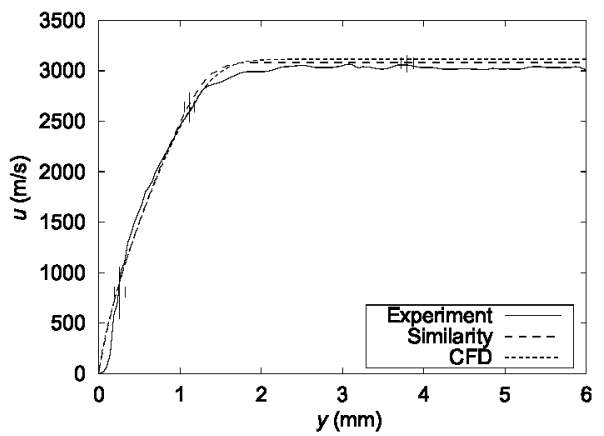

Figure 6: (a) Temperature and (b) velocity boundary layer profiles, obtained using the similarity method, ${ }^{19}$ CFDFASTRAN and experiment: $\gamma_{f r}=1.4$.

uncertainty, as shown in Fig. 6 (b). The change to the velocity profile caused by assuming a particular value of $\gamma_{f r}$ is small. This can be seen by the observation that the agreement between experiment and calculations is good for $\gamma_{f r}=1.3$, as shown in Fig. 7 (b).

The reason for the discrepancy in the peak temperature cannot be stated with certainty. It is possible that flow nonuniformity may have interfered with the temperature measurement, but the freestream rotational temperature agrees with the calculated values, and the large energy separation between the transitions makes the measurement insensitive to signal fluctuations. Flow nonuniformity also cannot explain the discrepancy in thermal boundary layer thickness between the experiment and the computations.

The most plausible explanation for the discrepancy between the measured and calculated peak temperatures is that part of the boundary layer may be in vibrational equilibrium, and would therefore have a ratio of specific heats closer to 1.3. The hottest part of the boundary layer also has velocities significantly lower than the freestream velocity. This increase in temperature and decrease in velocity could help that part of the flow to reach vibrational equilibrium. To test this hypothesis, a calculation of the characteristic vibrational relaxation distance $l_{v i b}$ was performed. The Landau-Teller formulation ${ }^{22}$ was used. Assuming that the relaxation is dominated by $\mathrm{N}_{2}-\mathrm{N}_{2}$ collisions, and using the freestream pressure with the measured temperature and velocity in the peak of the boundary layer, $l_{v i b}$ was calculated
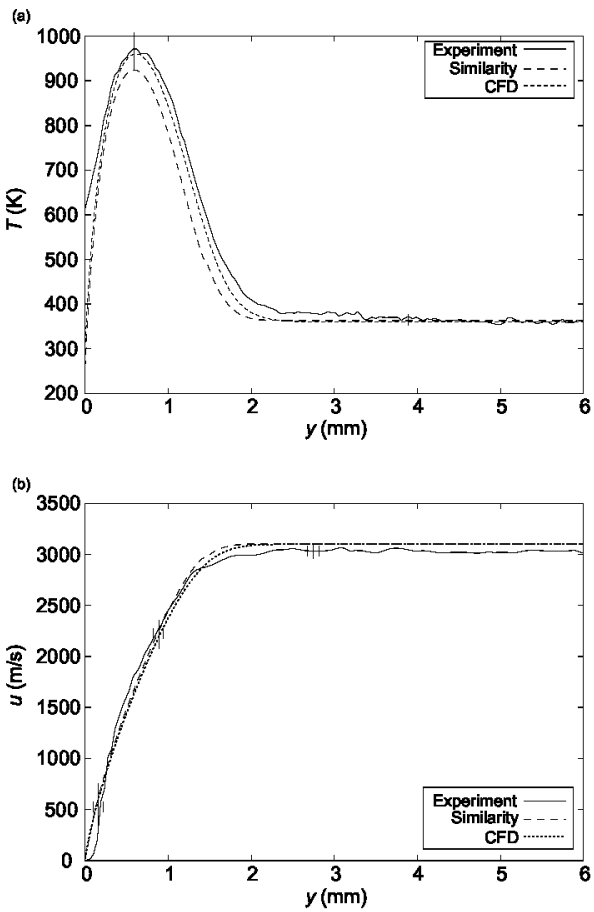

Figure 7: (a) Temperature and (b) velocity boundary layer profiles, obtained using the similarity method, ${ }^{19}$ CFDFASTRAN and experiment: $\gamma_{f r}=1.3$.

to be $160 \mathrm{~m}$. The length scale of the flat-plate experiments, $80 \mathrm{~mm}$, is much smaller than this, so the amount of vibrational relaxation should be negligible in the region near the temperature peak. The calculated relaxation distance for NO-NO collisions is $80 \mathrm{~mm}$, but this species only constitutes $1 \%$ of the fluid in the boundary layer and should have a negligible effect on measured rotational temperature.

Other possible effects which may have influenced the temperature profile include flow spillage around the model or catalytic re-combinations near the model surface. Neither of these explanations is convincing. If spillage were a significant problem, the velocity boundary layer would be thinner than the theoretical prediction, which is clearly not the case. Explanations relying upon absorption of energy by catalytic recombination are difficult to justify in a freestream composed of $99.6 \%$ diatomic species.

Another possible explanation is that the flat-plate model may have been misaligned in the test section, in spite of the alignment procedure descibed previously. The effect of a misalignment on the measured boundary layer properties can be investigated using the similar boundary-layer code. This calculation has been performed assuming a $3^{\circ}$ expansion angle, a measurable deviation from the nozzle axis. The effect of the misalignment was treated as a $3^{\circ}$ isentropic expansion of the freestream conditions, using the calculation technique in Ref. 23. This changed the freestream conditions, with the value of $\beta$ increasing from 0.252 for zero angle of attack to 0.376 . The peak of the calculated temperature distribution decreased from $1100 \mathrm{~K}$ to $1038 \mathrm{~K}$, 
improving the agreement with the experimental peak temperature of $970 \pm 45 \mathrm{~K}$. However, the expansion causes the freestream temperature to decrease from $360 \mathrm{~K}$, in good agreement with the measured temperature, to $305 \mathrm{~K}$. More importantly, the thermal and velocity boundary-layer thicknesses increase by $24 \%$ and $44 \%$ respectively. This much poorer agreement with the velocity measurements in particular makes model misalignment an unlikely explanation for the difference in measured and calculated peak temperatures.

\section{CONCLUSIONS}

A systematic comparison has been made between experimental temperature, surface heat flux and velocity measurements and two calculation methods for a laminar hypersonic boundary layer. The temperature measurements built upon previous measurements at slightly different conditions, ${ }^{7}$ while the velocity measurements are new, utilising a novel laser-based measurement technique. Both techniques allowed for direct measurements of the flow property of interest, without relying on theoretical flow calculation to infer distributions from surface data.

The temperature measurements were consistent with the previous measurements and showed qualitative agreement with both calculations. The peak temperature was $150 \mathrm{~K}$ lower than the calculated peak temperature, a fact which could not be adequately explained by measurement uncertainties. Good agreement occurred for calculations performed using $\gamma_{f r}=1.3$. This value for the ratio of specific heats is closer to that for equilibrium conditions, but the flowfield should be frozen, having $\gamma_{f r}=1.4$.

The most likely explanation for the disagreement between the calculated peak temperature and the measured value is that part of the boundary layer may be in vibrational equilibrium due to the higher temperatures and lower velocities in that portion of the flowfield. Although this is a plausible explanation for the discrepancy, the vibrational relaxation rates presented in Ref. 22 are too slow for relaxation to occur at the measured temperature and velocity conditions. It is conceivable that water or metallic contaminants in the flow could increase relaxation rates in the boundary layer. This hypothesis could be tested by performing measurements of vibrational temperature in the boundary layer, using vibrational PLIF thermometry or coherent anti-Stokes Raman spectroscopy.

The velocity measurements agreed to within experimental uncertainty with the calculated velocity profiles. The flow-tagging technique provided high-quality measurements with an uncertainty of $2 \%$, a great improvement on previous velocity measurement techniques used in freepiston shock tunnels. The technique shows great promise for velocity measurements in hypersonic flows.

The CFD-FASTRAN package provided a detailed model of the boundary layer flow, capturing both the leadingedge shock and the boundary layer flow, and converging to second-order accuracy. The local similarity calculation slightly underpredicted the boundary layer thickness when compared with the CFD-FASTRAN calculation. The most likely reason for this difference is that the displacement caused by the leading edge and other second-order effects were not accounted for. The local similarity calculation predicted the same trends as the CFD-FASTRAN calculation when parameters were varied, and convergence was nearly instantaneous. Its simplicity and speed make it an excellent choice for determining the effects on the boundary layer profile of varying the important flow parameters.

\section{ACKNOWLEDGMENTS}

The authors wish to thank Mr. Paul Walsh for his valuable technical assistance with the shock tunnel equipment. They thank Dr. Philip Palma for providing his LINUS code for calculations and for his valuable contributions to discussions of the PLIF technique. They also thank Dr. Glenn Diskin for providing the displacement fitting routines.

\section{References}

[1] East, R. A., Stalker, R. J., and Baird, J. P., "Measurements of Heat Transfer to a Flat Plate in a Dissociated High-enthalpy Laminar Air Flow," Journal of Fluid Mechanics, Vol. 97, No. 4, 1980, pp. 673-699.

[2] Mallinson, S. G., Gai, S. L., and Mudford, N. R., "The Boundary Layer on a Flat Plate in Hypervelocity Flow," The Aeronautical Journal, Vol. 100, No. 2, 1996, pp. 135-141.

[3] Baird, J. P., Lyons, P., and Gai, S. L., "Measurements of Density and Velocity Profiles in Non-equilibrium Laminar Hypersonic Boundary Layers in Air," 14th International Symposium on Shock Tubes and Waves, Sydney, Australia, 1983.

[4] Gross, K. P., M ${ }^{c}$ Kenzie, R. L., and Logan, P., "Measurements of Temperature, Density, Pressure and their Fluctuations in Supersonic Turbulence using LaserInduced Fluorescence," Experiments in Fluids, Vol. 5, 1987, pp. 372-380.

[5] Donohue, J. M. and $\mathrm{M}^{\mathrm{c}}$ Daniel Jr., J. C., "Computercontrolled Multiparameter Flowfield Measurements using Planar Laser-Induced Iodine Fluorescence," AIAA Journal, Vol. 34, No. 8, August 1996, pp. 1604 1611.

[6] O'Byrne, S., Hypersonic Laminar Boundary Layers and Near-Wake Flows, Ph.D. thesis, Australian National University, 2002.

[7] Palma, P. C., Mallinson, S. G., O'Byrne, S. B., Danehy, P. M., and Hillier, R., "Temperature Measurements in a Hypersonic Boundary layer using Planar Laser-induced Fluorescence," AIAA Journal, Vol. 38, No. 9, September 2000, pp. 1769-1772. 
[8] Danehy, P. M., O’Byrne, S., Houwing, A. F. P., Fox, J. S., and Smith, D. R., "Flow-Tagging Velocimetry for Hypersonic Flows using Fluorescence of Nitric Oxide," AIAA Journal, Vol. 41, No. 2, February 2003, pp. 263-271.

[9] Eckbreth, A. C., Laser Diagnostics for Combustion, Temperature and Species, Gordon and Breach, 2nd ed., 1996.

[10] Stalker, R. J., "A Study of the Free-Piston Shock Tunnel," AIAA Journal, Vol. 5, No. 12, May 1967, pp. 2160-2165.

[11] $\mathbf{M}^{\mathrm{C}}$ Intosh, M. K., "Computer Program for the Numerical Calculation of Frozen Equilibrium Conditions in Shock Tunnels," Tech. rep., Australian National University, 1968.

[12] Vardavas, I., "Modelling Reactive Gas Flows Within Shock Tunnels," Australian Journal of Physics, Vol. 37, 1984, pp. 157-177.

[13] He, Y. and Morgan, R. G., "Transition of Compressible High-enthalpy Boundary Layer Flow Over a Flat Plate," The Aeronautical Journal, Vol. 98, No. 2, 1994, pp. 25-33.

[14] Schultz, D. L. and Jones, T. V., "Heat Transfer Measurements in Short Duration Hypersonic Facilities," Tech. rep., AGARDograph AGARD-AG-165, 1973.

[15] Seitzman, J. M. and Hanson, R. K., "Planar Fluorescence Imaging in Gases," Instrumentation for Flows with Combustion, Academic Press Ltd, 1993.

[16] O'Byrne, S., Danehy, P. M., and Houwing, A. F. P., "Nonintrusive Temperature and Velocity Measurements in a Hypersonic Nozzle Flow," AIAA 20022917, 22nd AIAA Aerodynamic Measurement Technology and Ground Testing Conference, St Louis, USA, June 24-26, 2002.

[17] Palma, P. C., Laser-induced fluorescence imaging in free-piston shock tunnels, Ph.D. thesis, Australian National University, 1998.

[18] White, F. M., Viscous Fluid Flow, $\mathbf{M}^{\mathrm{c}}$ Graw-Hill, New York, 2nd ed., 1991.

[19] Haridas, A. J., Morphology of compressible laminar boundary layers, Master's thesis, University of Oklahoma, 1995.

[20] $\mathrm{M}^{\mathrm{C}}$ Intosh, M. K., "Free Stream Velocity Measurements in a High Enthalpy Shock Tunnel," Physics of Fluids, Vol. 14, No. 6, June 1971, pp. 1100-1102.

[21] Millikan, R. C. and White, D. R., "Systematics of Vibrational Relaxation," Journal of Chemical Physics, Vol. 39, No. 12, 1963, pp. 3209-3213.
[22] Vincenti, W. G. and Kruger, C. H., Introduction to Physical Gas Dynamics, Wiley, 1st ed., 1965.

[23] Rasmussen, M. L., Hypersonic Flow, Wiley Interscience, 1 st ed., 1994. 